IAU Colloquium 164: Radio Emission from Galactic and Extragalactic Compact Sources

ASP Conference Series, Vol. 144, 1998

J. A. Zensus, G. B. Taylor, \& J. M. Wrobel (eds.)

\title{
Some Theoretical Studies of Two Gamma-Ray Blazars: PKS 0528+134 and Mrk 421
}

\author{
S. J. Qian \& X. Z. Zhang \\ Beijing Astronomical Observatory, Beijing 100080, China
}

A. Witzel, T. P. Krichbaum, S. Britzen, \& A. Kraus

Max-Planck Institut für Radioastronomie, Bonn, Germany

\begin{abstract}
The high energy gamma-ray flares observed in PKS $0528+134$ are interpreted in terms of the external inverse Compton scattering (EICS) mechanism. The evolutional relationship between the gamma-ray flares and the associated mm-radio outbursts is investigated. The TeV/X-ray flare detected in May of 1994 from Mrk 421 is interpreted in terms of the SSC mechanism and it is shown that it may be due to the acceleration of relativistic electrons with an initially flat energy spectrum $\left(\mathrm{N}(\mathrm{E}) \propto E^{-s}\right.$ with $\left.\mathrm{s} \sim 1.5\right)$, rather than just a flattening of the high energy tail in the electron energy distribution of the source in the quiescent state.
\end{abstract}

\section{Evolutionary Relationship between Gamma-Ray Flares and mm- Radio Outbursts in PKS 0528+134}

Two gamma-ray flares were detected in PKS $0528+134$ by EGRET at epochs $\sim 1991.30$ and $\sim 1993.23$ (Michelson et al. 1994). The EICS mechanism (Sikora et al. 1994) may be appropriate to their interpretation. A model-fit to the overall spectral energy distributions $(\log (\mathrm{F} \nu)-\log \nu)$ is shown in Figure 1. The dashed and solid lines are for model-fitting the 1991.30 and 1993.23 events respectively. For the 1991.30 event, we show in figure 2 the low-frequency part of the synchrotron spectrum of the gamma-ray source derived from the model-fitting and the evolution track of the spectral turnover $F_{m}\left(\nu_{m}\right)$ (the solid line with two arrows) for the associated mm-radio outburst (Qian et al. 1996, Qian 1997). The three dashed-lines attached to the track are synchrotron spectra for three epochs. Shock-in-jet models suggest that flux density variations in blazars are caused by relativistic shocks propagating along jets from the innermost regions through to the outer regions, so that the spectral evolution tracks of synchrotron radiation of the gamma-ray sources should continuously transfer to those of the associated $\mathrm{mm}$-radio outbursts. Due to the lack of optical-IR and sub-mm monitoring data the spectral evolution track of the turn-over peak for the gamma-ray flaring source could not be derived. But Figure 2 suggests that this transition could occur between the decreasing branch of the track of the gamma-ray source (as the arrow denotes) and the rising branch of the track of the associated $\mathrm{mm}$-radio outburst. The details of this transition would need to be studied further.

\section{Acceleration of a Flat Electron Energy Spectrum and the $\mathrm{TeV} / \mathrm{X}$ - ray Flare in Mrk 421}

Macomb et al. (1995) reported that in Mrk 421 a TeV/X-ray flare was detected in May 1994 with little variations in the GeV, UV, IR and mm emissions. We propose two SSC-models, i.e., a two-component model and a single-component model, to investigate this phenomenon. In the two-component model the flare is assumed to be due to a flaring component which is independent from another 

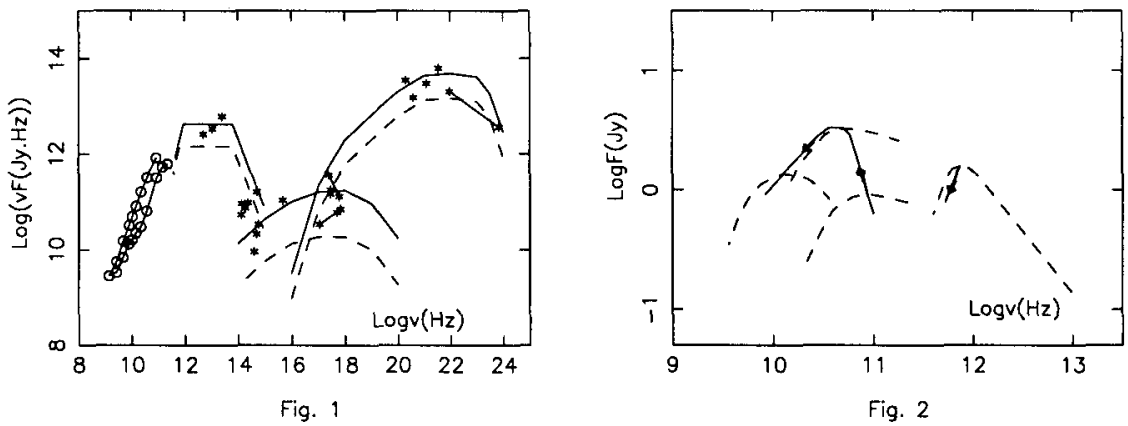

Fig. 2

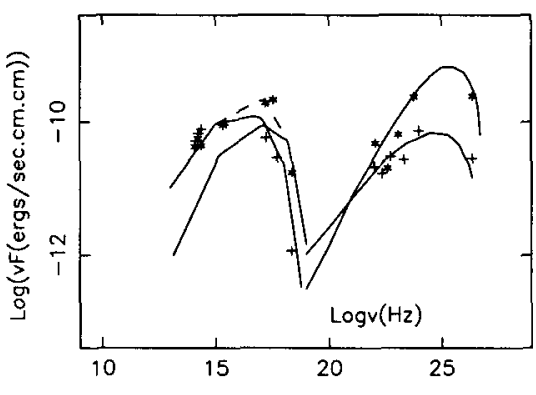

Fig. 3

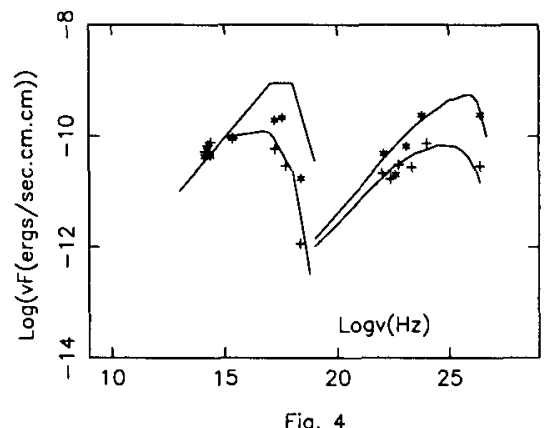

underlying quiescent component and related with the acceleration of relativistic electrons with an initially flat spectrum. In the single-component model the flare is assumed to be only due to the flattening of the high-energy tail of the electron energy distribution of the quiescent component at the highest energies. The model-fits for both the quiescent $(+)$ and flaring $(*)$ states are shown in figures 3 and 4 respectively. In the gamma-ray bands Klein-Nishina effects have been taken into account. Since the single-component model (fig.4) predicts a hard Xray flux larger than the observed flux, only the two-component model can well explain the properties of the $\mathrm{TeV} / \mathrm{X}$-ray flare. This may imply that the basic mechanism of particle acceleration in AGN produces flat energy distributions, consistent with observations of radio outbursts with spectral indices of $\sim 0.2-0.4$.

\section{References}

Macomb, D. J., et al. 1995. $A p J, 449$, L99-103.

Michelson, P. F., et al. 1994. in AIP Conf. Proc. 304: The Second Compton Symposium, eds. C. E. Fichtel, N. Gehrels, \& J. P. Norris (New York: Amer. Inst. of physics), 602-610.

Qian, S. J., et al. 1996, in ASP Conf. Ser. 100: Energy Transport in Radio Galaxies and Quasars, eds. P. E. Hardee, A. H. Bridle, \& J. A. Zensus (San Francisco : Astron. Soc. of the Pacific), 61-66.

Qian, S. J. 1997. Acta Astron. Sinica, 38, 239-249.

Sikora, M., Begelman, M. C. ,\& Rees, M. J. 1994. ApJ, 421, 153-162. 